\title{
Reliability and validity of the inertial sensor-based Timed "Up and Go" test in individuals affected by stroke
}

\author{
Seline Wüest, PhD; ${ }^{1 *}$ Fabien Massé, PhD $;^{2}$ Kamiar Aminian, PhD $;^{2}$ Roman Gonzenbach, MD, PhD ${ }^{3}$ Eling D. \\ de Bruin, PhD $^{\mathbf{1}}$ \\ ${ }^{1}$ Department of Health Sciences and Technology, Institute of Human Movement Sciences and Sport, ETH Zurich, \\ Zurich, Switzerland; ${ }^{2}$ Laboratory of Movement Analysis and Measurement, Ecole Polytechnique Fédérale de Laus- \\ anne, Lausanne, Switzerland; ${ }^{3}$ Department of Neurology, University Hospital Zurich, Zurich, Switzerland
}

\begin{abstract}
The instrumented Timed "Up and Go" test (iTUG) has the potential for playing an important role in providing clinically useful information regarding an individual's balance and mobility that cannot be derived from the original singleoutcome Timed "Up and Go" test protocol. The purpose of this study was to determine the reliability and validity of the iTUG using body-fixed inertial sensors in people affected by stroke. For test-retest reliability analysis, 14 individuals with stroke and 25 nondisabled elderly patients were assessed. For validity analysis, an age-matched comparison of 12 patients with stroke and 12 nondisabled controls was performed. Out of the 14 computed iTUG metrics, the majority showed excellent testretest reliability expressed by high intraclass correlation coefficients (range 0.431-0.994) together with low standard error of measurement and smallest detectable difference values. BlandAltman plots demonstrated good agreement between two repeated measurements. Significant differences between patients with stroke and nondisabled controls were found in 9 of 14 iTUG parameters analyzed. Consequently, these results warrant the future application of the inertial sensor-based iTUG for the assessment of physical deficits poststroke in longitudinal study designs.
\end{abstract}

Key words: assessment, balance, inertial sensors, instrumented Timed “Up and Go" test, mobility, motor function test, rehabilitation, stroke, test-retest reliability, validity.

\section{INTRODUCTION}

After a stroke, many individuals experience hemiparesis, which often leads to an impaired walking pattern with altered gait characteristics [1-7]. Specifically, hemiplegic gait is typically associated with a reduced gait speed [1-3,5,8], cadence [2,5,8], and stride length [2,8] and an increased left-right asymmetry during walking $[1,3,9]$ when compared with nondisabled, age-matched controls. Furthermore, it is often characterized by an increased stance phase duration [2]. Hemiparesis not only leads to gait impairments but may also affect balance and postural transfers (i.e., changing position from sitting to standing and vice versa), further impeding the patients' mobility and independence [6,10-12]. It is therefore a central goal of stroke rehabilitation to improve the

\footnotetext{
Abbreviations: $3-\mathrm{D}=$ three-dimensional, $\mathrm{CI}=$ confidence interval, ICC $=$ intraclass correlation coefficient, iTUG = instrumented Timed "Up and Go" test, LOA = limits of agreement, PD = Parkinson disease, $\mathrm{SD}=$ standard deviation, $\mathrm{SDD}=$ smallest detectable difference, SEM = standard error of measurement, TUG = Timed "Up and Go" test.

*Address all correspondence to Seline Wüest, PhD; Department of Health Sciences and Technology, Institute of Human Movement Sciences and Sport, Wolfgang-PauliStr 27, 8093 Zurich, ETH Zurich, Switzerland; +41-44632-3152. Email: seline27@gmx.ch http://dx.doi.org/10.1682/JRRD.2015.04.0065
} 
patient's independence and functional capacity by improving his or her mobility [13].

The Timed "Up and Go" test (TUG) is often used, with good reliability and validity, to evaluate balance and mobility in patients with stroke [14-17]. The TUG requires a person to rise from a chair, walk a distance of $3 \mathrm{~m}$ at a self-paced, comfortable speed, turn around, and return to the chair to sit down again. The total time for completion is recorded and used as a measure of mobility. However, although the TUG is commonly used to evaluate mobility poststroke, it has some drawbacks. First, it only uses the outcome parameter "time" and fails to detect other balance- and mobility-related parameters [18-19]. Second, although it consists of a number of consecutive tasks, it does not allow analysis of these tasks separately [18-19]. Recently, several researchers instrumented the TUG in an effort to overcome some of the drawbacks. For example, Vernon et al. used a Microsoft Kinect camera-based TUG method for analyzing the specific subcomponents of the test [20]. Furthermore, Zampieri et al. revealed the potential benefit of an instrumented TUG (iTUG) system using wearable inertial sensors while assessing individuals with Parkinson disease (PD) [19]. While 10 of the 21 gait and postural transition parameters that were identifiable with the iTUG showed significant differences between individuals with PD and nondisabled controls, the total test performance time was not diverging. Obviously, the iTUG seemed to exhibit a greater sensitivity than the conventional TUG in terms of mobility deficit detection in individuals with PD [18].

The recent use of body-fixed sensors suggests that they could serve as a tool for analyzing measures of physical functioning of patients [21-22]. An iTUG system using wearable sensors could potentially deliver more detailed and clinically relevant information on a patient with stroke's gait and mobility than the conventional TUG, which only reports the time to complete the task. To the best of our knowledge, no study exists applying an inertial sensor-based iTUG system in people affected by stroke. To be clinically useful, an assessment procedure must have a small measurement error to detect a real change and must be able to distinguish between subpopulations (e.g., patients with stroke in various stages and nondisabled controls). A test-retest difference in a patient with a value smaller than the standard error of measurement (SEM) is likely to be the result of "measurement noise" and is unlikely to be detected reliably in practice; a difference greater than the smallest real difference is highly likely (with 95\% confidence) to be a real difference
[23-25]. Another example of these statistics is the smallest detectable difference (SDD) $[24,26]$. The present study was conducted to assess the reliability and validity of inertial sensor-based iTUG metrics in patients with stroke and age-matched nondisabled control elderly patients.

\section{METHODS}

\section{Study Design}

The study was designed as an observational study in which all participants were tested by the same observer. Prior to participation, all participants were fully informed about the complete research protocol.

\section{Participants}

Participants were recruited on a voluntary basis through researchers from the Institute of Human Movement Sciences and Sport at ETH Zurich, Zurich, Switzerland, and by contacting physicians and therapists from the Rehabilitation Center Valens, Valens, Switzerland. There were two groups of participants. The first group included 14 patients at any stage after ischemic or hemorrhagic stroke aged over 18 yr. Participants were excluded from the study if any known comorbid disabilities other than stroke were present (e.g., musculoskeletal illness, cardiovascular disorders, or other neurologic diseases) that might have affected performance in the test procedures. The second group included 25 nondisabled by selfreport control participants aged over 65 yr who had no history of neurological, cardiovascular, or musculoskeletal pathologies. To be eligible for the study, individuals had to be able to walk unassisted for at least $15 \mathrm{~m}$. The use of one crutch for walking was accepted for inclusion. Individuals who were not able to give informed consent were excluded.

\section{Apparatus}

In total, eight body-fixed devices (Physilog, GaitUp; Lausanne, Switzerland) were placed on each participant's body. The sensor configuration described in the iTUG procedure by Salarian et al. [18]—one on each wrist, one on each shank, and one on the trunk-was complemented with one device on each foot and one on the back (lumbar 3) for further analysis. The sensor located on the patient's back was taped using hypoallergenic breathable straps to ensure firm attachment to the skin. Every other sensor device was firmly affixed to the patient using elastic straps. 
The devices recorded the signals from a calibrated inertial sensor (three-dimensional [3-D] accelerometer and 3-D gyroscope) to an onboard memory card at $500 \mathrm{~Hz}$ [27]. Before any processing, the inertial signals, sampled synchronously across devices, were resampled by software (MATLAB 2014a, MathWorks; Natick, Massachusetts) at $200 \mathrm{~Hz}$ as described in the original iTUG study [18].

\section{Measurement Protocol}

Each patient was first equipped with the wearable sensor set. Then, in accordance with Salarian et al. [18], we used an extended iTUG version with a $7 \mathrm{~m}$ walking distance. Thereby, more gait cycles were recorded than when using the original TUG protocol. All participants completed the testing session either in a gait laboratory of the Institute of Human Movement Sciences and Sport at ETH Zurich, Zurich, Switzerland, or in suitable locations at the Rehabilitation Center Valens, Valens, Switzerland.

The testing session included the following procedure: Initially, each participant was equipped with a set of sensors. Once the sensors had been attached on the appropriate body positions, they performed the first iTUG measurement session composed of three repeated iTUG trials (measurement session 1). Between each trial, a $30 \mathrm{~s}$ rest period was given. After completing the three iTUG trials, the participants were instructed to relax for 15 min while removing the sensors from their bodies. Then, the sensors were reattached to participants' bodies and the same protocol was repeated (measurement session 2). Each testing session was recorded with a video camera, allowing researchers later inspection if necessary.

In order to get sufficient information about the assessment tool's reliability and validity characteristics, we identified the relative reliability by using the intraclass correlation coefficient (ICC) and the absolute reliability to identify a real improvement by calculating (a) the SEM for groups of subjects, (b) the limits of agreement (LOA) for a single person, and (c) the SDD, which also reveals the limits for the real change for a single person. Additionally, we identified the discriminatory capabilities on the iTUG metrics by comparing stroke patients with nondisabled age-matched controls.

\section{Data Analysis}

Based on algorithms described elsewhere [18-19,2829], different parameters of gait and postural transition during iTUG performance were measured.

- iTUG Trial
- iTUG total duration: total duration of the iTUG trial in seconds.

- Sit-to-Walk Metrics

- Sit-to-walk duration: duration of the sit-to-walk transition in seconds.

- Peak sit-to-walk velocity: maximum angular trunk velocity in degrees per second during the sit-to-walk transition.

- Gait Metrics

- Gait cadence: walking cadence in number of steps per minute; normalized to participants' height.

- Gait stance phase: stance phase as a percentage of gait cycle time.

- Gait limp phase: difference between initial and terminal double support phase as a percentage of gait cycle time.

- Gait velocity: walking speed in meters per second; normalized to participants' height.

- Gait stride length: distance in meters between two consecutive foot falls at the moments of initial contact; normalized to participants' height.

- Gait peak swing velocity: maximum angular shank velocity in degrees per second during one stride.

- Gait asymmetry: symmetry ratio related to the swing phase performed by each leg calculated with the formula: symmetry ratio $=\mid 1-$ [(limb with lower value) / (limb with higher value)]|.

- Turning Metrics

- Turning duration: duration of $180^{\circ}$ turn in seconds.

- Peak turning velocity: maximum angular trunk velocity in degrees per second while turning.

- Turn-to-Sit Metrics

- Turn-to-sit duration: duration of the turn-to-sit transition in seconds.

- Peak turn-to-sit velocity: maximum angular trunk velocity in degrees per second during the turn-to-sit transition.

The reliability and validity assessments apply to the averaged values of three iTUG trials, as opposed to the original TUG in which the outcome is based on a single measure following a practice trial. For data analysis, we used the mean value of the iTUG total duration across the three iTUG trials performed in measurement session 1 and in measurement session 2 . The median of the metrics of the straight walking gait, the transitions, and the turns across the three iTUG trials performed in both measurement sessions was used to eliminate possible outliers. 
Excepting the parameter "gait asymmetry," which is based on the swing phase performed by each leg, the average value of both legs was used.

\section{Statistical Analysis}

Descriptive statistical analysis was carried out to describe the study population. The one-sample KolmogorovSmirnov test, skewness, and kurtosis were used to test normality of the data. The primary test-retest reliability calculations were based on the entire study population. A reliability subanalysis including only the patients with stroke was performed separately. The reliability subanalysis incorporated the five gait iTUG metrics: cadence, stance phase, velocity, stride length, and asymmetry. For assessing differences between stroke patients and nondisabled elderly patients (age-matched), two groups were created (stroke and nondisabled control) and compared with the paired $t$-test or the nonparametric equivalent where appropriate. All statistical analyses were performed using SPSS version 21.0 software (IBM Corporation; Armonk, New York). The critical $\alpha$-level was set at $p \leq 0.05$.

\section{Reliability}

Several statistical methods of assessing test-retest reliability were performed. Heteroscedasticity was tested by calculating the square value of Pearson correlation coefficient $\left(r^{2}\right)$ between the absolute difference and the mean of each pair of measurements. If values of $r^{2}$ are greater than 0.1 , then the data are heteroscedastic [30]. The ICC with the 95 percent confidence interval (CI) was used as an estimate of relative reliability [30]. The ICC is commonly used to determine the consistency or reproducibility between repeated measurements and to assess the SEM [31]. In this article, the ICC IC $_{1, k}$ one-way analysis of variance was considered because the same device and same participants tested by the sole rater were used for assessing test-retest reliability. Furthermore, the two measurement sessions performed during the study were separated by a time period of only $15 \mathrm{~min}$. Therefore, we assumed that participants' gait patterns would not have changed over this time.

Interpretation of the ICC values was according to Shrout and Fleiss [32], in which values of $>0.75$ indicate excellent reliability, 0.75 to 0.40 indicate fair to good reliability, and $<0.40$ indicate poor reliability. Because the ICC score depends greatly on the range of values in the analyzed sample [30] and is not able to provide infor- mation about the accuracy for a specific individual, the SEM and the SDD for each parameter were calculated. The SEM indicates a real improvement in the group of individuals and was assessed using the equation: $\mathrm{SEM}=\mathrm{SD} \sqrt{1-\mathrm{ICC}}$ in which SD represents the sample standard deviation [30]. The SDD can be used as an indicator for assessing real change beyond measurement error in a single person. It was derived from the SEM through $1.96 \times \sqrt{2} \times$ SEM [26]. The SEM and SDD can be expressed as percentages that are independent of the units of measurement and, therefore, suitable to compare the amount of random error between measurement parameters: SEM percent $=[(\mathrm{SEM} /$ mean of the two measurements $) \times 100]$ and SDD percent $=[($ SDD $/$ mean of the two measurements) $\times 100$ ].

Discrepancies between the measurements were also investigated by performing Bland-Altman 95 percent LOA analysis. It expresses the degree of error proportional to the mean. The Bland-Altman method includes a scatter plot providing information about the degree of error (measurement 1 -measurement 2) proportional to the mean of the two measurements with 95 percent LOA (mean difference $\pm 1.96 \times$ SD of the difference) [24,33-34].

\section{Validity}

The paired $t$-test and the Wilcoxon signed-rank test were performed to examine differences between stroke patients and the age-matched nondisabled controls, depending on normality of data. Group mean values of the iTUG metrics are expressed as mean \pm SD. Effect sizes are presented as Pearson correlation coefficient $(r)$, which can be calculated from the $t$-statistics converted into $r$-statistics by Equation 1:

$$
r=t^{2} /\left(t^{2}+d f\right)
$$

or from the Z-value using Equation 2:

$$
r=\frac{Z}{\sqrt{N}},
$$

in which $d f$ represents the degrees of freedom, $Z$ represents the approximation of the observed difference in terms of the standard normal distribution, and $N$ represents the total number of observations [35]. The effect size magnitude of $r=0.1$ indicates a small, $r=0.3$ a medium, and $r=0.5$ a large effect [35]. The results of the first measurement session were used for analysis. Because the gait parameters walking speed, stride length, 
and cadence are related to someone's body height, these parameters were normalized to height (actual parameter value / height) [36].

\section{RESULTS}

A total of 39 participants were enrolled in the study, of which 25 were nondisabled elderly participants. The characteristics of this study population are described in Table 1. Because the two groups differed in mean age, an age-matched comparison of 12 patients with stroke and 12 age-matched nondisabled controls was performed for validity analysis. The characteristics of those participants are presented in Table 2.

\section{Reliability}

Out of the 14 analyzed iTUG metrics, the ICCs of 12 variables showed excellent test-retest reliability $\left(\mathrm{ICC}_{1, k}=0.855-0.994\right)$. Only the two sit-to-walk parameters, "duration" and "peak sit-to-walk velocity," reported a lower relative reliability coefficient $\left(\mathrm{ICC}_{1, k}=\right.$ 0.431 and $\mathrm{ICC}_{1, k}=0.674$, respectively). The ICC values with corresponding 95 percent CI are reported together with the SEM and SEM percent, the SDD and SDD percent, and the 95 percent LOA in Table 3. Values of $r^{2}$ based on the absolute differences between measurement session 1 and measurement session 2 and the mean value of both measurement sessions were for all iTUG parameters below 0.1 , indicating no evidence of heteroscedastic- ity. Bland-Altman plots graphically support homoscedasticity in all iTUG variables analyzed (see the Appendix, available online only, for the BlandAltman plots). Eleven of the 14 analyzed iTUG parameters showed low SEM and SEM percent values (0.220\%-7.109\%), and low SDD and SDD percent values (0.659\%-19.706\%). High SEM and SEM percent values (15.819\%-20.600\%), and high SDD and SDD percent values (43.848\%$57.134 \%$ ) were found for both sit-to-walk parameters and for the gait parameter related to the limp phase.

The reliability subanalysis of the gait iTUG metrics "cadence," "stance phase," "velocity," "stride length," and "asymmetry" revealed similar results as those based on the entire study population (Table 4); for all five iTUG metrics measured, excellent test-retest reliability $\left(\mathrm{ICC}_{1, k}=0.958-0.991\right)$ with concomitant low SEM and SEM percent (0.431\%-2.701\%), and low SDD and SDD percent values (1.292\%-7.481\%) were found.

\section{Validity}

The results of the validity analysis are presented in Table 5. Considering the total time duration needed for iTUG completion, there was a statistically significant difference between the two groups. The patients with stroke required more time to complete the iTUG than the agematched nondisabled controls. Among the 13 computed iTUG subcomponent parameters, 8 showed significant between-group differences.

Table 1.

Demographic data of the entire study population.

\begin{tabular}{|c|c|c|c|c|c|}
\hline Demographic & $\begin{array}{l}\text { Patients with } \\
\text { Stroke }\end{array}$ & $\begin{array}{l}\text { Nondisabled Elderly } \\
\text { Controls }\end{array}$ & Total & $p$-Value & $r$-Value \\
\hline Participants $(n)$ & 14 & 25 & 39 & - & - \\
\hline Sex (female/male) & $2 / 12$ & $17 / 8$ & $19 / 20$ & - & - \\
\hline Height (cm), mean \pm SD (range) & $175.4 \pm 6.0(166.0-186.0)$ & $167.7 \pm 9.2(152.0-189.0)$ & $170.4 \pm 8.9(152.0-189.0)$ & $0.008^{*}$ & 0.174 \\
\hline Walking Assistance, $n$ (\%) & $5(35.7)$ & $0(0.0)$ & $5(12.8)$ & - & - \\
\hline Affected Side (right/left) & $8 / 6$ & - & - & - & - \\
\hline
\end{tabular}


Table 2.

Demographic data of the age-matched study population.

\begin{tabular}{|c|c|c|c|c|c|}
\hline Demographic & $\begin{array}{l}\text { Patients with } \\
\text { Stroke }\end{array}$ & $\begin{array}{l}\text { Nondisabled Elderly } \\
\text { Controls }\end{array}$ & Total & $p$-Value & $r$-Value \\
\hline Participants $(n)$ & 12 & 12 & 24 & - & - \\
\hline Age (yr), mean \pm SD (range) & $67.5 \pm 6.2(57.0-76.0)$ & $71.2 \pm 3.0(66.0-76.0)$ & $69.3 \pm 5.1(57.0-76.0)$ & 0.08 & 0.134 \\
\hline Sex (female/male) & $0 / 12$ & $9 / 3$ & $9 / 15$ & - & - \\
\hline Height (cm), mean \pm SD (range) & $176.4 \pm 5.7(168.0-186.0)$ & $168.9 \pm 9.5(157.0-189.0)$ & $172.7 \pm 8.6(157.0-189.0)$ & $0.03^{*}$ & 0.200 \\
\hline Weight (kg), mean \pm SD (range) & $85.2 \pm 14.0(70.0-114.0)$ & $75.3 \pm 12.4(60.2-101.1)$ & $80.3 \pm 13.9(60.2-114.0)$ & 0.08 & 0.131 \\
\hline BMI $\left(\mathrm{kg} / \mathrm{m}^{2}\right)$, mean \pm SD (range) & $27.3 \pm 4.0(22.6-35.2)$ & $26.3 \pm 2.6(21.7-30.4)$ & $26.8 \pm 3.3(21.7-35.2)$ & 0.45 & 0.026 \\
\hline Walking Assistance $n(\%)$ & $5(41.7)$ & $0(0.0)$ & $5(20.8)$ & - & - \\
\hline Affected Side (right/left) & $7 / 5$ & - & - & - & - \\
\hline
\end{tabular}

Table 3.

Reliability of the instrumented Timed "Up and Go" test metrics based on the entire study population (patients with stroke and nondisabled elderly controls).

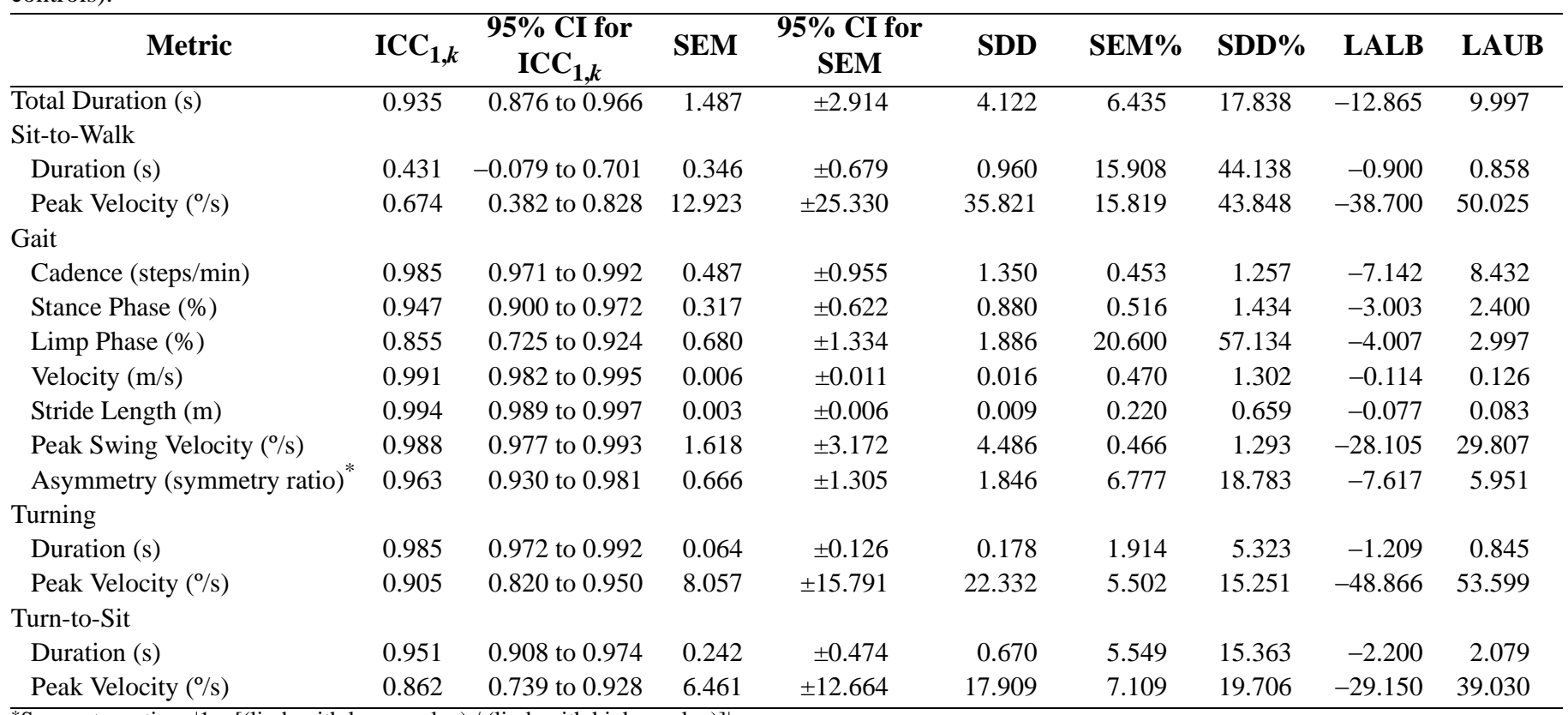

${ }^{*}$ Symmetry ratio $=\mid 1-[($ limb with lower value) $/$ (limb with higher value) $] \mid$.

$\mathrm{CI}=$ confidence interval, $\mathrm{ICC}_{1, k}=$ intraclass correlation coefficient (one-way analysis), LALB $=$ limits of agreement lower boundary, LAUB $=$ limits of agreement upper boundary, SDD = smallest detectable difference, SEM = standard error of measurement.

\section{DISCUSSION}

This study was performed to analyze inertial sensorbased iTUG metrics regarding (1) test-retest reliability in a group composed of patients with stroke patients and nondisabled elderly controls and (2) the ability to discriminate between individuals with stroke and age-matched nondisabled controls. The present study revealed that all but two of the computed iTUG metrics reached ICCs $>0.75$, which indicates excellent relative reliability. Both variables that did not achieve excellent relative reliability values were related to the iTUG subcomponent "sit-to-walk." Comparable results found in this study were reported by Salarian et al. [18], who performed the inertial sensor-based iTUG in patients with PD and nondisabled participants. Three out of the four major iTUG subcomponents, "sit-to-stand," 
Table 4.

Reliability of the instrumented Timed "Up and Go" test metrics based on patients with stroke for gait.

\begin{tabular}{lccccccccc}
\hline \multicolumn{1}{c}{ Metric } & ICC $_{\mathbf{1 , k} \boldsymbol{k}}$ & $\begin{array}{c}\text { 95\% CI for } \\
\text { ICC }_{\mathbf{1 , k}}\end{array}$ & SEM & $\begin{array}{c}\mathbf{9 5 \% ~ C I ~ f o r ~} \\
\text { SEM }\end{array}$ & SDD & SEM\% & SDD\% & LALB & LAUB \\
\hline Cadence (steps/min) & 0.983 & 0.948 to 0.994 & 0.488 & \pm 0.957 & 1.354 & 0.512 & 1.421 & -3.603 & 11.081 \\
Stance Phase (\%) & 0.958 & 0.873 to 0.986 & 0.327 & \pm 0.641 & 0.907 & 0.522 & 1.448 & -4.198 & 2.059 \\
Velocity (m/s) & 0.985 & 0.955 to 0.995 & 0.007 & \pm 0.014 & 0.020 & 0.746 & 2.131 & -0.057 & 0.173 \\
Stride Length (m) & 0.991 & 0.973 to 0.997 & 0.005 & \pm 0.011 & 0.015 & 0.431 & 1.292 & -0.087 & 0.136 \\
Asymmetry & 0.981 & 0.942 to 0.994 & 0.464 & \pm 0.910 & 1.285 & 2.701 & 7.481 & -6.810 & 6.078 \\
\hline
\end{tabular}

$\mathrm{CI}=$ confidence interval, $\mathrm{ICC}_{1, k}=$ intraclass correlation coefficient (one-way analysis), LALB $=$ limits of agreement lower boundary, LAUB $=$ limits of agreement upper boundary, SDD = smallest detectable difference, SEM = standard error of measurement.

Table 5.

Differences between groups (patients with stroke and nondisabled elderly controls) for instrumented Timed "Up and Go" test metrics.

\begin{tabular}{|c|c|c|c|c|c|c|}
\hline Metric & $\begin{array}{l}\text { Patients with } \\
\text { Stroke }\end{array}$ & $\begin{array}{c}\text { Nondisabled } \\
\text { Elderly Controls }\end{array}$ & Mean Diff \pm SD & $\begin{array}{l}\text { 95\% CI for } \\
\text { Mean Diff }\end{array}$ & $p$-Value & $r$-Value \\
\hline Total Duration (s) & $27.66 \pm 12.73$ & $18.19 \pm 1.84$ & $9.477 \pm 11.86$ & 1.941 to 17.013 & $0.002^{*}$ & 0.577 \\
\hline \multicolumn{7}{|l|}{ Sit-to-Walk } \\
\hline Duration (s) & $2.17 \pm 0.47$ & $2.10 \pm 0.29$ & $0.073 \pm 0.57$ & -0.289 to 0.435 & 0.95 & 0.021 \\
\hline Peak Velocity ( $\%$ s) & $69.77 \pm 26.48$ & $97.81 \pm 20.33$ & $28.043 \pm 34.81$ & 5.925 to 50.162 & $0.02^{*}$ & 0.414 \\
\hline \multicolumn{7}{|l|}{ Gait } \\
\hline Cadence (steps/min) & $54.6 \pm 10.9$ & $68.9 \pm 7.4$ & $14.278 \pm 12.80$ & 6.144 to 22.411 & $0.005^{*}$ & 0.544 \\
\hline Stance Phase (\%) & $62.20 \pm 4.19$ & $61.01 \pm 1.39$ & $1.187 \pm 4.81$ & -1.868 to 4.243 & 0.85 & 0.048 \\
\hline Limp Phase (\%) & $3.64 \pm 2.87$ & $2.80 \pm 1.86$ & $0.841 \pm 3.98$ & -1.689 to 3.370 & 0.85 & 0.048 \\
\hline Velocity $(\mathrm{m} / \mathrm{s})$ & $0.57 \pm 0.17$ & $0.85 \pm 0.06$ & $0.282 \pm 0.19$ & 0.160 to 0.404 & $0.001^{*}$ & 0.609 \\
\hline Stride Length (m) & $0.69 \pm 0.16$ & $0.88 \pm 0.05$ & $0.187 \pm 0.18$ & 0.070 to 0.303 & $0.002^{*}$ & 0.577 \\
\hline Peak Swing Velocity ( $\%$ s) & $297.56 \pm 59.24$ & $391.38 \pm 34.43$ & $93.824 \pm 80.06$ & 42.957 to 144.690 & $0.001^{*}$ & 0.609 \\
\hline Asymmetry & $15.60 \pm 11.28$ & $6.42 \pm 3.39$ & $9.179 \pm 13.65$ & 0.503 to 17.854 & 0.09 & 0.352 \\
\hline \multicolumn{7}{|l|}{ Turning } \\
\hline Duration (s) & $5.15 \pm 2.83$ & $2.16 \pm 0.35$ & $2.994 \pm 2.78$ & 1.230 to 4.757 & $<0.001^{*}$ & 0.624 \\
\hline Peak Velocity (\%/s) & $95.34 \pm 24.47$ & $180.13 \pm 28.44$ & $84.788 \pm 36.97$ & 61.297 to 108.278 & $<0.001^{*}$ & 0.852 \\
\hline \multicolumn{7}{|l|}{ Turn-to-Sit } \\
\hline Duration (s) & $5.98 \pm 3.63$ & $3.17 \pm 1.19$ & $2.812 \pm 2.76$ & 1.060 to 4.564 & $<0.001^{*}$ & 0.625 \\
\hline Peak Velocity ( $\%$ s) & $97.51 \pm 36.81$ & $88.71 \pm 19.42$ & $8.804 \pm 37.73$ & -15.168 to 32.776 & 0.73 & 0.080 \\
\hline
\end{tabular}

Note: $p$-value for between-groups comparison; $r$-value, effect size, calculated according to $r=Z \sqrt{N}$ and $r=t^{2} /(t 2+d f) ; r=0.1$ : small effect, $r=0.3$ : medium effect, $r=0.5$ large effect.

*Significant between-group differences $\left(p_{\text {between }} \leq 0.05\right)$ calculated with paired $t$-tests and Wilcoxon signed-rank test.

Mean Diff = mean difference [(mean of the group with higher value $)-($ mean of the group with lower value $)$, SD $=$ standard deviation of the difference, $\mathrm{CI}=$ confidence interval.

"steady-state gait," "turning," and "turn-to-sit," showed good to excellent reliability for most of their contributing metrics [18]. In line with our findings, the parameters "cadence" and "stance phase" have emerged as two of the most reliable iTUG variables with ICCs greater than 0.90 . Regarding the subcomponents "turning” and "turn-to-sit," there was consensus that the parameter "duration" seems to be the most reliable. Moreover, in accordance with our study, Salarian et al. revealed the sit-to-stand subcomponent as the least reliable part of the iTUG [18].
Besides high ICCs, a measurement tool should exhibit small measurement errors and be able to identify real changes in the group and in single individuals [37]. The absolute reliability analysis performed in this study corroborates the good relative reliability of the inertial sensor-based iTUG metrics. Specifically, low SEM and SEM percent values together with low SDD and SDD percent values were found in 11 of the total computed 14 iTUG parameters, which indicates good precision of the measurement tool. 
Except for a few outliers, most of the value differences between the two repeated measurements fell within the 95 percent LOA in the Bland-Altman plots and were well distributed around zero (see the Appendix, available online only), demonstrating good agreement between the data of repeated measurements.

On average, the patients improved between the first and the second measurement (Bland-Altman plots in the Appendix, available online only). This is likely a learning effect between the first and second measurement. Performing an initial test trial prior to the actual measurement may have prevented this learning effect.

Several studies described the typical "hemiplegic gait” poststroke with a decreased walking velocity, cadence, and stride length [1-3,5,8]. Moreover, an increase in stance phase duration and left-right asymmetry seems to be a characteristic feature of the gait of patients with stroke [1-3,9]. Accordingly, in this study those five iTUG metrics were highlighted by a separate reliability analysis for which the stroke group was separated from the nondisabled elderly controls (Table 4). The subanalysis showed similar relative test-retest reliability values as found in the entire study population. Specifically, excellent ICCs above 0.75 were achieved. Furthermore, all five iTUG metrics in the subanalysis demonstrated good absolute test-retest reliability expressed by small SEM and SEM percent values together with small SDD and SDD percent values. These findings warrant further investigations of the iTUG in larger samples of people with stroke and, thus, further exploring the clinical relevance of the system.

In clinical settings, the assessment of mobility and balance in people with stroke is important for several reasons, including to accurately perform a diagnosis, to plan the treatment method for each individual patient, and to adequately evaluate the rehabilitation effectiveness. The iTUG system has the potential for playing an important role in providing clinically useful information by objectively assessing a patient with stroke's balance and mobility. This highlights its potential relevance for application in clinical practice.

Out of the 14 analyzed iTUG metrics, 9 showed a significant difference between patients with stroke and nondisabled controls (Table 5). Among the variables that were significantly different between the two groups, all demonstrated high effect size values close to 0.50 and larger. In contrast, smaller effect sizes ranging from 0.02 to 0.35 were measured for those five parameters that did not differ between the two groups. Specifically, there were no significant differences in the duration required for completing the iTUG subcomponent "sit-to-walk," in the percentage values related to "stance phase" and "limp phase” of gait cycle time during steady-state gait, in the left-right gait asymmetry, and in the maximum angular trunk velocity during turn-to-sit transition.

From previous studies, we have indication that stroke leads to walking- and balance-related limitations and negatively affects patients' functional mobility. Research by Ng and Hui-Chan indicates that there was a significant difference in the TUG performance duration between patients with chronic stroke and nondisabled elderly patients [14]. In line with our study, the patients with stroke needed more time to finish the TUG procedure. Furthermore, significantly higher values for walking speed, cadence, and step length in favor of the nondisabled individuals were found. Regarding the variable "stance time," Ng and Hui-Chan revealed a discrepancy between the two groups, but only when the outcomes based on the unaffected sides of patients with stroke were considered [14]. In our study, no separate body siderelated analysis was done and, therefore, no specific information regarding the paretic and nonparetic side was available. Hence, we recommend that this should be part of future studies. Olney and Richards stated an increase of the stance phase proportion within the gait cycle in patients with stroke [38]. In the present study, however, no significant difference was found between patients with stroke and age-matched nondisabled controls. The inconsistency between the studies may be related to differences in stroke characteristics and severity.

Gait symmetry represents an indicator of normal walking [39]. Because stroke often results in unilateral symptoms contralateral to the infarct, normalization of gait symmetry may be an indicator of gait recovery. For gait symmetry evaluation, various metrics can be considered. According to the literature, the most common symmetry metrics used for gait assessment are swing time, single support time, pelvic and/or trunk movement, and ground reaction forces [39-40]. Patterson et al. concluded that the parameter "swing time" is suitable to analyze gait symmetry poststroke and, therefore, highly recommended [40]. Hence, this study focused on the gait variable "swing phase" for symmetry measure. Our results of the gait symmetry evaluation are, however, at odds with the findings of Patterson et al., who reported a more asymmetric gait pattern in patients with stroke when compared 
with nondisabled individuals. In the current study, no significant asymmetry difference was found [40]. Nonetheless, the effect size for gait asymmetry exceeded the 0.3 level and showed, consequently, a medium effect. One possible reason for this might be the rather small sample of people with stroke with insufficient between-subjects variance. Future studies should, therefore, repeat our study design in larger samples of patients with stroke in different poststroke recovery phases.

Another interesting parameter to be considered for gait analysis of patients with stroke is arm swing. Indication exists that the asymmetries frequently seen in gait parameters poststroke also affect upper-body movements, resulting in asymmetric arm-swing patterns [41]. The hemiparetic gait of patients with stroke is often associated with an adducted arm with no or limited arm swing on the affected side. A normalization of the arm swing on the affected side might be a parameter for recovery of normal gait. Zampieri et al. identified the iTUG parameter "peak arm swing velocity" as one of the most sensitive deficits in early PD [19]. It might be that the parameter "arm swing" is also of clinical relevance poststroke. Future research that includes arm-swing parameters as outcome variable is warranted.

\section{LIMITATIONS}

Some limitations of this study should be considered. One was the lack of detailed information regarding the severity, type, and anatomical lesion location(s) of the stroke. Further studies are needed that focus on specific subgroups in the stroke population (e.g., subacute or chronic stroke, first or recurrent stroke) to substantiate our findings of the inertial sensor-based iTUG application in clinical settings. Moreover, the pertinence of using elderly patients to test the iTUG may be questioned. However, untrained elderly patients normally show both balance and gait impairments [42-46], which should render them an adequate population for assessing reliability as well. The sex distribution in our sample may be another limitation given the sex imbalance between the patients with stroke and the age-matched nondisabled controls; all the stroke patients were male, whereas only 3 of 12 nondisabled controls were male. This may be a source of bias confounding the results. Furthermore, sex disparity in stroke prevalence persists, with women being more affected than men [47], making it especially impor- tant to test reliability in women in future studies. A further limitation of this study was the small sample size in relation to reliability studies. An adequate sample size for the assessment of the agreement parameter, based on a general guideline by Altman [48], lies around 50. The sample size of 14 patients with stroke and 25 nondisabled elderly individuals we used is, however, a realistic group size to find first estimates for the assumed relation between stroke and functionally important tasks as measured with the iTUG and to gather results in preliminary data as a basis for further examinations including larger samples. Furthermore, the reliability subanalysis has to be interpreted with caution; other than the data used for the primary reliability analysis based on the entire study population, the subanalysis data were not checked for heteroscedasticity.

\section{CONCLUSIONS}

Excellent test-retest reliability was found for most of the iTUG metrics measured, and the inertial sensor-based iTUG is able to distinguish patients with stroke from nondisabled controls. These findings suggest that the inertial sensor-based iTUG measures are useful to assess functional mobility in patients with stroke. However, the study should be repeated with a larger group of patients to investigate its discriminatory capabilities between different subgroups of stroke patients.

\section{ACKNOWLEDGMENTS}

\section{Author Contributions:}

Study concept and design: S. Wüest, K. Aminian, E. D. de Bruin. Acquisition of data: S. Wüest, F. Massé, R. Gonzenbach, E. D. de Bruin.

Analysis and interpretation of data: S. Wüest, F. Massé, E. D. de Bruin.

Drafting of manuscript: S. Wüest, E. D. de Bruin.

Critical revision of manuscript for important intellectual content:

R. Gonzenbach, E. D. de Bruin.

Administrative, technical, or material support: F. Massé, K. Aminian, R. Gonzenbach.

Financial Disclosures: The authors have declared that no competing interests exist.

Funding/Support: This material was based on work partially supported by the REWIRE project (http://sites.google.com/site/projectrewire/), funded by the European Commission under the FP7 framework (contract 287713). 
Additional Contributions: We thank Dr. Jürg Kesselring, Gabriela Wyttenbach, and Susanne Müller, Clinics of Valens, for support with organization and recruiting of patients.

Institutional Review: Ethical approval for this study was granted by the local ETH Zurich Ethics Committee (protocol number EK 2012N-32) and the cantonal Ethics Committee of the Canton of St. Gallen (protocol number EKSG 12/002/1B). All participants signed an informed consent form.

Participant Follow-Up: The authors have no plans to notify the study subjects of the publication of this article because of a lack of contact information.

\section{REFERENCES}

1. Hornby TG, Straube DS, Kinnaird CR, Holleran CL, Echauz AJ, Rodriguez KS, Wagner EJ, Narducci EA. Importance of specificity, amount, and intensity of locomotor training to improve ambulatory function in patients poststroke. Top Stroke Rehabil. 2011;18(4):293-307. [PMID:21914594] http://dx.doi.org/10.1310/tsr1804-293

2. Dickstein R, Dunsky A, Marcovitz E. Motor imagery for gait rehabilitation in post-stroke hemiparesis. Phys Ther. 2004;84(12):1167-77. [PMID:15563257]

3. Hsu AL, Tang PF, Jan MH. Analysis of impairments influencing gait velocity and asymmetry of hemiplegic patients after mild to moderate stroke. Arch Phys Med Rehabil. 2003;84(8):1185-93. [PMID:12917858] http://dx.doi.org/10.1016/S0003-9993(03)00030-3

4. Puh U, Baer GD. A comparison of treadmill walking and overground walking in independently ambulant stroke patients: A pilot study. Disabil Rehabil. 2009;31(3):202-10. [PMID:18608434]

http://dx.doi.org/10.1080/09638280801903039

5. von Schroeder HP, Coutts RD, Lyden PD, Billings E Jr, Nickel VL. Gait parameters following stroke: A practical assessment. J Rehabil Res Dev. 1995;32(1):25-31. [PMID:7760264]

6. Chen G, Patten C, Kothari DH, Zajac FE. Gait deviations associated with post-stroke hemiparesis: Improvement during treadmill walking using weight support, speed, support stiffness, and handrail hold. Gait Posture. 2005;22(1):57-62. [PMID:15996593] http://dx.doi.org/10.1016/j.gaitpost.2004.06.008

7. Boudarham J, Roche N, Pradon D, Bonnyaud C, Bensmail D, Zory R. Variations in kinematics during clinical gait analysis in stroke patients. PLoS ONE. 2013;8(6):e66421. [PMID:23799100]

http://dx.doi.org/10.1371/journal.pone.0066421

8. Lennon S. Gait re-education based on the Bobath concept in two patients with hemiplegia following stroke. Phys Ther. 2001;81(3):924-35. [PMID:11268157]
9. Dietz V. Quadrupedal coordination of bipedal gait: Implications for movement disorders. J Neurol. 2011;258(8): 1406-12. [PMID:21553270]

http://dx.doi.org/10.1007/s00415-011-6063-4

10. Barreca S, Sigouin CS, Lambert C, Ansley B. Effects of extra training on the ability of stroke survivors to perform an independent sit-to-stand: A randomized controlled trial. J Geriatr Phys Ther. 2004;27(2):59-64. http://dx.doi.org/10.1519/00139143-200408000-00004

11. Ada L, Westwood P. A kinematic analysis of recovery of the ability to stand up following stroke. Aust J Physiother. 1992;38(2):135-42. [PMID:25025645] http://dx.doi.org/10.1016/S0004-9514(14)60558-4

12. Cameron DM, Bohannon RW, Garrett GE, Owen SV, Cameron DA. Physical impairments related to kinetic energy during sit-to-stand and curb-climbing following stroke. Clin Biomech (Bristol, Avon). 2003;18(4):332-40. [PMID:12689783] http://dx.doi.org/10.1016/S0268-0033(03)00023-8

13. Wüest S, van de Langenberg R, de Bruin ED. Design considerations for a theory-driven exergame-based rehabilitation program to improve walking of persons with stroke. Eur Rev Aging Phys Act. 2014;11(2):119-29. Erratum in Eur Rev Aging Phys Act. 2014;11(2):142.

[PMID:25309631] http://dx.doi.org/10.1007/s11556-013-0136-6

14. Ng SS, Hui-Chan CW. The Timed Up \& Go Test: Its reliability and association with lower-limb impairments and locomotor capacities in people with chronic stroke. Arch Phys Med Rehabil. 2005;86(8):1641-47. [PMID:16084820] http://dx.doi.org/10.1016/j.apmr.2005.01.011

15. Kitsos G, Harris D, Pollack M, Hubbard IJ. Assessments in Australian stroke rehabilitation units: A systematic review of the post-stroke validity of the most frequently used. Disabil Rehabil. 2011;33(25-26):2620-32.

[PMID:21554012] http://dx.doi.org/10.3109/09638288.2011.575526

16. Faria CD, Teixeira-Salmela LF, Nadeau S. Predicting levels of basic functional mobility, as assessed by the Timed "Up and Go" Test, for individuals with stroke: Discriminant analyses. Disabil Rehabil. 2013;35(2):146-52. [PMID:22671699] http://dx.doi.org/10.3109/09638288.2012.690497

17. Sibley KM, Straus SE, Inness EL, Salbach NM, Jaglal SB. Balance assessment practices and use of standardized balance measures among Ontario physical therapists. Phys Ther. 2011;91(11):1583-91. [PMID:21868613] http://dx.doi.org/10.2522/ptj.20110063

18. Salarian A, Horak FB, Zampieri C, Carlson-Kuhta P, Nutt JG, Aminian K. iTUG, a sensitive and reliable measure of mobility. IEEE Trans Neural Syst Rehabil Eng. 2010; 
18(3):303-10. [PMID:20388604]

http://dx.doi.org/10.1109/TNSRE.2010.2047606

19. Zampieri C, Salarian A, Carlson-Kuhta P, Aminian K, Nutt JG, Horak FB. The instrumented Timed Up and Go Test: Potential outcome measure for disease modifying therapies in Parkinson's disease. J Neurol Neurosurg Psychiatry. 2010;81(2):171-76. [PMID:19726406] http://dx.doi.org/10.1136/jnnp.2009.173740

20. Vernon S, Paterson K, Bower K, McGinley J, Miller K, Pua YH, Clark RA. Quantifying individual components of the Timed Up and Go using the Kinect in people living with stroke. Neurorehabil Neural Repair. 2015;29(1):48-53. [PMID:24743227]

21. de Bruin ED, Hartmann A, Uebelhart D, Murer K, Zijlstra W. Wearable systems for monitoring mobility-related activities in older people: A systematic review. Clin Rehabil. 2008;22(10-11):878-95. [PMID:18955420] http://dx.doi.org/10.1177/0269215508090675

22. Allet L, Knols RH, Shirato K, de Bruin ED. Wearable systems for monitoring mobility-related activities in chronic disease: A systematic review. Sensors (Basel). 2010; 10(10):9026-52. [PMID:22163393]

http://dx.doi.org/10.3390/s101009026

23. Hartmann A, Knols R, Murer K, de Bruin ED. Reproducibility of an isokinetic strength-testing protocol of the knee and ankle in older adults. Gerontology. 2009;55(3):259-68. [PMID:18997454] http://dx.doi.org/10.1159/000172832

24. de Bruin ED, Hubli M, Hofer P, Wolf P, Murer K, Zijlstra W. Validity and reliability of accelerometer-based gait assessment in patients with diabetes on challenging surfaces. J Aging Res. 2012;2012;954378. [PMID:22900182] http://dx.doi.org/10.1155/2012/954378

25. Sole G, Hamrén J, Milosavljevic S, Nicholson H, Sullivan SJ. Test-retest reliability of isokinetic knee extension and flexion. Arch Phys Med Rehabil. 2007;88(5):626-31. [PMID:17466732] http://dx.doi.org/10.1016/j.apmr.2007.02.006

26. de Bruin ED, Rozendal RH, Stüssi E. Reliability of phasevelocity measurements of tibial bone. Phys Ther. 1998; 78(11):1166-74. [PMID:9806621]

27. Ferraris F, Grimaldi U, Parvis M. Procedure for effortless in-field calibration of three-axial rate gyro and accelerometers. Sensors and Materials. 1995;7(5):311-30.

28. Aminian K, Najafi B, Büla C, Leyvraz PF, Robert P. Spatio-temporal parameters of gait measured by an ambulatory system using miniature gyroscopes. J Biomech. 2002;35(5):689-99. [PMID:11955509] http://dx.doi.org/10.1016/S0021-9290(02)00008-8

29. Salarian A, Russmann H, Vingerhoets FJ, Dehollain C, Blanc Y, Burkhard PR, Aminian K. Gait assessment in Parkinson's disease: Toward an ambulatory system for long- term monitoring. IEEE Trans Biomed Eng. 2004;51(8): 1434-43. [PMID:15311830]

http://dx.doi.org/10.1109/TBME.2004.827933

30. Atkinson G, Nevill AM. Statistical methods for assessing measurement error (reliability) in variables relevant to sports medicine. Sports Med. 1998;26(4):217-38.

[PMID:9820922]

http://dx.doi.org/10.2165/00007256-199826040-00002

31. Huang SL, Hsieh CL, Wu RM, Tai CH, Lin CH, Lu WS. Minimal detectable change of the Timed "Up \& Go" Test and the Dynamic Gait Index in people with Parkinson disease. Phys Ther. 2011;91(1):114-21. [PMID:20947672] http://dx.doi.org/10.2522/ptj.20090126

32. Shrout PE, Fleiss JL. Intraclass correlations: Uses in assessing rater reliability. Psychol Bull. 1979;86(2):420-28.

[PMID:18839484] http://dx.doi.org/10.1037/0033-2909.86.2.420

33. Euser AM, Dekker FW, le Cessie S. A practical approach to Bland-Altman plots and variation coefficients for log transformed variables. J Clin Epidemiol. 2008;61(10):978-82. [PMID:18468854] http://dx.doi.org/10.1016/j.jclinepi.2007.11.003

34. Ageberg E, Bennell KL, Hunt MA, Simic M, Roos EM, Creaby MW. Validity and inter-rater reliability of mediolateral knee motion observed during a single-limb mini squat. BMC Musculoskelet Disord. 2010;11:265.

[PMID:21080945] http://dx.doi.org/10.1186/1471-2474-11-265

35. Field A. Discovering statistics using SPSS. Thousand Oaks (CA): Sage Publications; 2009.

36. Senden R, Meijer K, Heyligers IC, Savelberg HH, Grimm B. Importance of correcting for individual differences in the clinical diagnosis of gait disorders. Physiotherapy. 2012;98(4):320-24. [PMID:23122438]

http://dx.doi.org/10.1016/j.physio.2011.06.002

37. Hadzic V, Ursej E, Kalc M, Dervisevic E. Reproducibility of shoulder short range of motion in isokinetic and isometric strength testing. J Exerc Sci Fit. 2012;10(2):83-89. http://dx.doi.org/10.1016/j.jesf.2012.10.005

38. Olney SJ, Richards C. Hemiparetic gait following stroke. Part I: Characteristics. Gait Posture. 1996;4(2):136-48. http://dx.doi.org/10.1016/0966-6362(96)01063-6

39. Hodt-Billington C. Measures of symmetry in gait. Methodological principles and clinical choices [dissertation]. [Bergen (Norway)]: University of Bergen; 2012.

40. Patterson KK, Gage WH, Brooks D, Black SE, McIlroy WE. Evaluation of gait symmetry after stroke: A comparison of current methods and recommendations for standardization. Gait Posture. 2010;31(2):241-46. [PMID:19932621] http://dx.doi.org/10.1016/j.gaitpost.2009.10.014

41. Hodt-Billington C, Helbostad JL, Moe-Nilssen R. Should trunk movement or footfall parameters quantify gait asymmetry in chronic stroke patients? Gait Posture. 2008;27(4): 
552-58. [PMID:17897830]

http://dx.doi.org/10.1016/j.gaitpost.2007.07.015

42. Bohannon RW. Comfortable and maximum walking speed of adults aged 20-79 years: Reference values and determinants. Age Ageing. 1997;26(1):15-19. [PMID:9143432] http://dx.doi.org/10.1093/ageing/26.1.15

43. Chetta A, Zanini A, Pisi G, Aiello M, Tzani P, Neri M, Olivieri D. Reference values for the 6-min walk test in healthy subjects 20-50 years old. Respir Med. 2006; 100(9): 1573-78. [PMID:16466676] http://dx.doi.org/10.1016/j.rmed.2006.01.001

44. de Bruin ED, van Het Reve E, Murer K. A randomized controlled pilot study assessing the feasibility of combined motor-cognitive training and its effect on gait characteristics in the elderly. Clin Rehabil. 2013;27(3):215-25. [PMID:22865831] http://dx.doi.org/10.1177/0269215512453352

45. Troosters T, Gosselink R, Decramer M. Six minute walking distance in healthy elderly subjects. Eur Respir J. 1999; 14(2):270-74. [PMID:10515400] http://dx.doi.org/10.1034/j.1399-3003.1999.14b06.x

46. Winter DA, Patla AE, Frank JS, Walt SE. Biomechanical walking pattern changes in the fit and healthy elderly. Phys Ther. 1990;70(6):340-47. [PMID:2345777]
47. Towfighi A, Markovic D, Ovbiagele B. Persistent sex disparity in midlife stroke prevalence in the United States. Cerebrovasc Dis. 2011;31(4):322-28. [PMID:21212663] http://dx.doi.org/10.1159/000321503

48. Altman DG. Practical statistics for medical research. London (UK): Chapman and Hall; 1991.

Submitted for publication April 14, 2015. Accepted in revised form August 21, 2015.

This article and any supplementary material should be cited as follows:

Wüest S, Massé F, Aminian K, Gonzenbach R, de Bruin ED. Reliability and validity of the inertial sensor-based Timed "Up and Go" test in individuals affected by stroke. J Rehabil Res Dev. 2016;53(5):599-610.

http://dx.doi.org/10.1682/JRRD.2015.04.0065

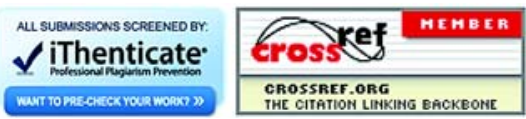

